\title{
Total flavonoids of Rhizoma drynariae ameliorate steroid-induced avascular necrosis of the femoral head via the PI3K/AKT pathway
}

\author{
WENXUE LV ${ }^{1 *}$, MINGXIU YU ${ }^{2 *}$, QINGYI YANG ${ }^{1}$, PENG KONG $^{1}$ and BING YAN ${ }^{1}$ \\ Departments of ${ }^{1}$ Orthopaedics and ${ }^{2}$ Special Inspection, The Affiliated Hospital of Shandong University of \\ Traditional Chinese Medicine, Lixia, Jinan, Shandong 250014, P.R. China
}

Received August 21, 2020; Accepted February 16, 2021

DOI: $10.3892 / \mathrm{mmr} .2021 .11984$

\begin{abstract}
Steroid-induced avascular necrosis of the femoral head (SANFH) is a common orthopaedic disease that is difficult to treat. The present study investigated the effects of total flavonoids of Rhizoma drynariae (TFRD) on SANFH and explored its underlying mechanisms. The SANFH rat model was induced by intramuscular injection of lipopolysaccharides and methylprednisolone. Osteoblasts were isolated from the calvariae of neonatal rats and then cultured with dexamethasone (Dex). TFRD was used in vitro and in vivo, respectively. Haematoxylin and eosin staining was used to assess the pathological changes in the femoral head. Terminal deoxynucleotidyl transferase-mediated deoxyuridine triphosphate nick end labelling assay and flow cytometry were conducted to detect apoptosis of osteoblasts. The 2',7'-dichlorofluorescein-diacetate staining method was used to detect reactive oxygen species (ROS) levels in osteoblasts and the 3-(4,5-dimethylthiazol-2-yl)-2,5-diphenyltetrazolium bromide assay was used to detect osteoblast proliferation. The expression of caspase-3, Bax, Bcl-2, VEGF, runt-related transcription factor 2 (RUNX2), osteoprotegerin (OPG), osteocalcin $(\mathrm{OCN})$, receptor activator of nuclear factor $\mathrm{\kappa B}$ ligand (RANKL) and phosphoinositide 3-kinase (PI3K)/AKT
\end{abstract}

Correspondence to: Dr Bing Yan, Department of Orthopaedics, The Affiliated Hospital of Shandong University of Traditional Chinese Medicine, 16369 Jingshi Road, Lixia, Jinan, Shandong 250014, P.R. China

E-mail: yanbing128@126.com

*Contributed equally

Abbreviations: SANFH, steroid-induced avascular necrosis of the femoral head; Dex, dexamethasone; TFRD, total flavonoids from Rhizoma drynariae; ROS, reactive oxygen species; RUNX2, runt-related transcription factor 2; OPG, osteoprotegerin; OCN, osteocalcin; RANKL, receptor activator of nuclear factor kappa B ligand; PI3K, phosphoinositide 3-kinase

Key words: total flavonoids of Rhizoma drynariae, steroid-induced avascular necrosis of the femoral head, osteoblast, PI3K/AKT pathway, apoptosis pathway related-proteins were detected via western blotting. It was found that TFRD reduced the pathological changes, inhibited apoptosis, increased the expression of VEGF, RUNX2, OPG and OCN, decreased RANKL expression and activated the PI3K/AKT pathway in SANFH rats. TFRD promoted proliferation, inhibited apoptosis and reduced ROS levels by activating the PI3K/AKT pathway in osteoblasts. In conclusion, TFRD protected against SANFH in a rat model. In addition, TFRD protected osteoblasts from Dex-induced damage through the PI3K/AKT pathway. The findings of the present study may contribute to find an effective treatment for the management of SANFH.

\section{Introduction}

Osteonecrosis of the femoral head (ONFH) is one of the most common diseases affecting the hip joint, resulting in severe pain or joint disability $(1,2)$. Steroid-induced avascular necrosis of the femoral head (SANFH) is the most common type of ONFH (3). If patients with SANFH are not treated properly, the femoral head may collapse, resulting in hip function damage and disability $(4,5)$. Although conservative treatment and surgery have been used as SANFH treatment, there is no recognized effective therapy thus far. Therefore, it is important to explore the exact pathogenesis of SANFH and find an ideal treatment for SANFH.

Medicinal herbs are used to treat various diseases in Japan, Korea, China, other Asian areas and now all over the world (6). Chinese herb-derived active components provide valuable compounds for new drug candidate development (7). Rhizoma drynariae is one of the herbs from Davallia (L.) Sm., which comprises phenolic acids, triterpenes and flavonoids (8). Total flavonoids of Rhizoma drynariae (TFRD) is a herbaceous component extracted from the drained root of Rhizoma drynariae and the effective monomer of TFRD includes neoeriocitrin, naringenin and naringin $(9,10)$. This compound stimulates bone formation or inhibits bone resorption by modulating signalling in pathways involving bone morphogenetic protein in bone metabolism, Wnt/ $\beta$-catenin, receptor activator of nuclear factor $\kappa \mathrm{B}$ ligand (RANKL)/RANK and cathepsin K (a cysteine protease) (8). In addition, naringin serves as an active compound of TFRD to inhibit bone loss and promote bone repair in rats with glucocorticoid-induced ONFH via 
the AKT/Bad pathway (11). However, the effects of TFRD on SANFH have not been reported.

The phosphoinositide 3-kinase (PI3K)/AKT pathway serves an important role in several basic cellular processes including survival, proliferation, growth and differentiation $(12,13)$. A study has shown that the phosphatase and tensin homolog regulate angiogenesis in human pancreatic cancer cells through the PI3K/AKT/VEGF pathway (14). Zhang et al (15) reported that exosomes could enhance bone regeneration by activating the PI3K/AKT pathway. Hu et al (16) found that ginsenoside $\mathrm{Rg} 1$ protected rat bone marrow stem cell apoptosis induced by hydrogen peroxide through the PI3K/AKT pathway. Hence, the present study hypothesized that the PI3K/AKT pathway might be associated with the pathogenesis of SANFH. However, there is little research on the role of this pathway in SANFH.

The present study investigated the function of TFRD on rats with SANFH, including pathological changes of the femoral head, apoptosis of bone cells and the PI3K/AKT pathway. In addition, the function of TFRD in osteoblasts was investigated. The present study might provide useful information on the pathogenesis of SANFH and support the further clinical application of TFRD.

\section{Materials and methods}

Animals. A total of 24 male Sprague-Dawley rats (12 weeks old; 250-300 g; Liaoning Changsheng Biotechnology Co., Ltd.) were used in the present study. All rats were housed under standard laboratory conditions (12-h light/dark cycle, $24-25^{\circ} \mathrm{C}$ and $50-55 \%$ humidity ) and provided free access to food and water. The experiments were performed in accordance with the National Institutes of Health Guide for the Care and Use of Laboratory Animals (8th edition) (17) and approved by the Institutional Animal Care and Use Committee of the Affiliated Hospital of Shandong University of Traditional Chinese Medicine (approval no. 2019-58).

Grouping and animal model establishment. All rats were randomized into control, SANFH, or SANFH+TFRD groups ( $n=8$ each). TFRD was purchased from Beijing Qihuang Pharmaceutical Manufacturing Co., Ltd. (trade name: Qiang gu capsuled, drug approval number: Z20030007). Based on the instructions of Qiang gu capsule, clinical usage (0.75 g•day for adults) and the Meeh-Rubner equation (18) of dose conversion, $20 \mathrm{mg} / \mathrm{kg}$ dosage was chosen for administration to rats by intraperitoneal injection daily. A previous study reported that rats were administered with Rhizoma drynariae extract at dose of $20 \mathrm{mg} / \mathrm{kg}$ body weight once daily (19). The SANFH model was induced by intramuscular injection of lipopolysaccharides (LPS) and methylprednisolone (MPS). Briefly, rats in the SANFH and SANFH+TFRD groups were given two doses of $20 \mu \mathrm{g} / \mathrm{kg}$ LPS (Escherichia coli 055: B5; Sigma-Aldrich; Merck KGaA) on days 0 and 1 (twice at $24 \mathrm{~h}$ intervals) and administered $40 \mathrm{mg} / \mathrm{kg}$ MPS (Pfizer, Inc.) on days 3, 4 and 5 (three times after a 24-h interval). Rats in the control group were injected with normal saline. Additionally, rats in the SANFH+TFRD group were intraperitoneally injected with $20 \mathrm{mg} / \mathrm{kg} /$ day TFRD every day, while the remainder of the rats were injected with normal saline. The time interval for all injections was $24 \mathrm{~h}$. All rats were weighed weekly.
Subsequently, four weeks after the final MPS injection, all rats were sacrificed by intraperitoneal overdose anaesthesia with pentobarbital sodium (240 mg/kg, Sigma-Aldrich; Merck $\mathrm{KGaA}$ ) and femoral heads were harvested for subsequent tests.

Haematoxylin and eosin staining. The femur specimens were fixed in $4 \%$ paraformaldehyde for $48 \mathrm{~h}$ at $4^{\circ} \mathrm{C}$ and then decalcified with $10 \%$ ethylenediaminetetraacetic acid for 4-6 weeks at room temperature. After complete decalcification, the specimens were dehydrated in graded ethanol, embedded in paraffin and cut into $4-\mu \mathrm{m}$ sections. Thereafter, the sections were dewaxed with xylene and then gradually dehydrated via gradient ethanol. The sections were stained with haematoxylin solution for $5 \mathrm{~min}$ at $37^{\circ} \mathrm{C}$ followed by eosin solution for $2 \mathrm{~min}$ at $37^{\circ} \mathrm{C}$. Finally, the sections were dehydrated with gradient ethanol, washed three times using xylene and then mounted in neutral gum. The histopathological changes were examined under a light microscope (Leica Microsystems $\mathrm{GmbH}$ ) by two independent authors. Three sections were obtained from each femoral head and the percentage of empty lacunae was assessed at x100 magnification in five randomly selected fields per section. The diagnosis of ONFH was established on the basis of the pathological features of empty bone lacuna numbers and surrounding necrotic bone marrow tissues.

Terminal deoxynucleotidyl transferase deoxyuridine triphosphate nick end labelling (TUNEL) assay. TUNEL assay was used to detect the apoptosis rate of osteoblasts using a Situ Cell Death Detection kit (Nanjing Jiancheng Bioengineering Institute) according to the manufacturer's protocol. Following routine dewaxing and dehydration, the sections were digested with pepsin ( 0.25\%-0.5\% hydrochloric acid solution) for $25 \mathrm{~min}$ at room temperature, incubated with $50 \mu 1$ TUNEL reaction mixture for $1 \mathrm{~h}$ at $37^{\circ} \mathrm{C}$ and then incubated with $50 \mu \mathrm{l}$ of peroxidase for $30 \mathrm{~min}$ at $37^{\circ} \mathrm{C}$. Thereafter, the sections were dyed with 3,3'-diaminobenzidine reagent and stained with hematoxylin for $2 \mathrm{~min}$ at room temperature. Cells with brown nuclei were considered as TUNEL-positive cells and were further assessed under a fluorescence microscope (Olympus Corporation). The apoptosis rate of osteoblasts was defined as the proportion of the number of TUNEL-positive cells to the total number of cells.

Western blot analysis. Total protein was extracted from the osteoblasts and tissues of the femoral head using radioimmunoprecipitation assay buffer containing protease and phosphatase inhibitors (Abcam). A bicinchoninic acid (BCA) kit (Thermo Scientific, Inc.) was used to measure the total protein concentration. Equal amounts of protein ( $30 \mu \mathrm{g} / \mathrm{lane})$ were loaded on $10 \%$ sodium dodecyl sulphate-polyacrylamide gel electrophoresis and then transferred onto polyvinylidene fluoride membranes (Pall Life Sciences). Thereafter, the membranes were blocked with 5\% non-fat milk (Sigma-Aldrich; Merck KGaA) in Tris-buffer saline containing $0.1 \%$ Tween-20 for $1 \mathrm{~h}$ at room temperature and then incubated with the following primary antibodies overnight at $4^{\circ} \mathrm{C}$ : Anti-caspase-3 (cat. no. 14220; 1:1,000), anti-Bax (cat. no. 14796; 1:1,000), anti-RUNX2 (cat. no. 12556; 1:1,000), anti-phosphorylated (p-)PI3K (cat. no. 17366; 1:1,000), anti-p-AKT (cat. no. 4060; 1:1,000), anti-AKT (cat. no. 4691; 1:1,000) and anti-glyceraldehyde 
3-phosphate dehydrogenase (internal parameter; cat. no. 5174; 1:1,000 all from Cell Signaling Technology, Inc.; anti-Bcl-2 (ab196495; 1:1,000) and anti-OCN (ab13418; 1:1,000), from Abcam; and anti-VEGF (sc-7269; 1:1,000), anti-OPG (sc-390518; 1:1,000), anti-RANKL (sc-377079; 1:1,000) and anti-PI3K (sc-1637; 1:1,000) from Santa Cruz Biotechnology, Inc. The membranes were then incubated with corresponding horseradish peroxidase-conjugated secondary antibodies (1:1,000, Wuhan Boster Biological Technology Co., Ltd.) for $1 \mathrm{~h}$ at room temperature. Finally, the labelled proteins were treated with enhanced chemiluminescence (ECL) solution (Pierce; Thermo Fisher Scientific, Inc.) and the protein bands were analysed using ImageJ software, version 1.8.0 (National Institutes of Health).

Cell culture and treatment. Osteoblasts were isolated from calvariae of neonatal rats as previously described (20). Briefly, heads of 2-day-old neonatal rats were cut off, soaked in phosphate-buffered saline (PBS; HyClone; Cytiva) and the calvariae were then dissected out. Thereafter, the calvariae were cut into pieces, incubated with $1 \%$ trypsin (Gibco; Thermo Fisher Scientific, Inc.) for $15 \mathrm{~min}$ at $37^{\circ} \mathrm{C}$ and then incubated with PBS containing $0.25 \%$ collagenase (Type I; Sigma-Aldrich; Merck KGaA) for $60 \mathrm{~min}$ at $37^{\circ} \mathrm{C}$. Following centrifugation at $300 \mathrm{x}$ g for $10 \mathrm{~min}$ at $37^{\circ} \mathrm{C}$, the osteoblasts were obtained and cultured in $\alpha$-minimal essential medium containing $12 \%$ foetal bovine serum and antibiotics (Gibco; Thermo Fisher Scientific, Inc.) at $37^{\circ} \mathrm{C}$ with $5 \% \mathrm{CO}_{2}$.

Osteoblasts were treated with different concentrations of dexamethasone (Dex; 0, 10 ${ }^{-6}, 10^{-7}$ and $10^{-8} \mathrm{M}$; Sigma-Aldrich; Merck KGaA) for $0,24,48$ and $72 \mathrm{~h}$ at $37^{\circ} \mathrm{C}$ or TFRD $(0,0.1$, $0.5,2.5,12.5$ and $62.5 \mu \mathrm{g} / \mathrm{ml}$ ) for $48 \mathrm{~h}$ at $37^{\circ} \mathrm{C}$ to estimate the dosage effects on cell proliferation. Osteoblasts were divided into control (without treatment), Dex (osteoblasts treated with $10^{-6} \mathrm{M}$ Dex for $48 \mathrm{~h}$ at $37^{\circ} \mathrm{C}$ ), Dex+TFRD [osteoblasts pre-treated with $12.5 \mu \mathrm{g} / \mathrm{ml}$ TFRD (the maximum concentration of TFRD without cytotoxicity on osteoblasts) for $2 \mathrm{~h}$ at $37^{\circ} \mathrm{C}$ and then incubated with $10^{-6} \mathrm{M}$ Dex for $48 \mathrm{~h}$ at $37^{\circ} \mathrm{C}$ ], Dex+TFRD+LY294002 group (osteoblasts pre-treated with $12.5 \mu \mathrm{g} / \mathrm{ml}$ TFRD for $2 \mathrm{~h}$ at $37^{\circ} \mathrm{C}$ and $10 \mu \mathrm{M} \mathrm{LY} 294002$ [an inhibitor of PI3K/AKT pathway; Beyotime Institute of Biotechnology] for $1 \mathrm{~h}$ at $37^{\circ} \mathrm{C}$ and then incubated with $10^{-6} \mathrm{M}$ Dex for $48 \mathrm{~h}$ at $37^{\circ} \mathrm{C}$ ). Following treatment, osteoblasts were collected for further experiments.

MTT assay. Osteoblast proliferation was assessed using the MTT assay. Briefly, osteoblasts $\left(2.0 \times 10^{4}\right.$ cells $\left./ \mathrm{ml}\right)$ were seeded onto 96-well plates and exposed to TFRD, Dex and LY294002 as detailed in the previous experiment, respectively. Thereafter, osteoblasts were incubated with $20 \mu \mathrm{l}$ MTT solution $\left(5 \mathrm{mg} / \mathrm{ml}\right.$, Beyotime Institute of Biotechnology) at $37^{\circ} \mathrm{C}$ for $4 \mathrm{~h}$ and then incubated with $150 \mu \mathrm{l}$ dimethyl sulfoxide. Finally, optical density (OD) was measured at $490 \mathrm{~nm}$ with a spectrophotometric plate reader (Bio-Rad Laboratories, Inc.). The experiments were repeated three times.

Flow cytometry. The Annexin V-fluorescein isothiocyanate (FITC) early apoptosis detection kit (Cell Signaling Technology, Inc.) was used to analyse osteoblast apoptosis. Briefly, osteoblasts were incubated with Annexin V-FITC
(10 $\mu \mathrm{l})$ and propidium iodide $(5 \mu \mathrm{l})$ for $30 \mathrm{~min}$ without light at room temperature. The apoptosis rate of osteoblasts (including early and late apoptotic cells) was measured using a BD FACSCanto II flow cytometer (Becton, Dickinson and Company).

Reactive oxygen species (ROS) production assay. The ROS level in osteoblasts was measured using a dichlorofluorescein-diacetate (DCFH-DA) dye (Sigma-Aldrich; Merck $\mathrm{KGaA})$. Briefly, osteoblasts $\left(3 \times 10^{3}\right.$ cells per well) were seeded into 24-well plates and exposed to different treatments as detailed in the previous experiments. The osteoblasts were then collected, washed with PBS and incubated with $10 \mu \mathrm{M}$ DCFH-DA for $30 \mathrm{~min}$ at $37^{\circ} \mathrm{C}$ without light. Fluorescence intensity was measured via flow cytometry.

Statistical analysis. All data were presented as the mean \pm standard deviation and analysed using GraphPad Prism 7.0 software (GraphPad Software, Inc.). Significant differences among three or more groups were assessed by one-way analysis of variance (ANOVA) with Tukey's post hoc test. $\mathrm{P}<0.05$ was considered to indicate a statistically significant difference.

\section{Results}

TFRD reduces the pathological changes of the femoral head and inhibits apoptosis of the necrotic zone in SANFH rats. In the present study, LPS and MPS were used to establish a rat model of SANFH. To investigate the influence of TFRD on SANFH rats, rats were intraperitoneally injected with TFRD. During the experimental period, all rats survived and there were no significant differences in the mean body weights among the control, SANFH and SANFH+TFRD groups ( $\mathrm{P}>0.05$; Fig. 1A). Haematoxylin and eosin staining was used to assess the pathological changes in the femoral head. As shown in Fig. 1B, bone structure was intact in the control group, while there were many empty bone lacunae and broken trabeculae in the SANFH group. In addition, the percentage of empty lacuna in the SANFH+TFRD group was lower compared with the $\mathrm{SANFH}$ group $(\mathrm{P}<0.05)$. TUNEL assay was conducted to detect apoptosis of the bone cells of the femoral head. As presented in Fig. 1C, the apoptosis rate in the SANFH and SANFH+TFRD groups was higher compared with the control group $(\mathrm{P}<0.01)$, however treatment with TFRD reduced the apoptosis rate compared with the SANFH group $(\mathrm{P}<0.05)$. In addition, the expression of apoptosis-related proteins (caspase-3, Bax and Bcl-2) was detected via western blotting. The results showed that the expression of caspase- 3 and Bax in the SANFH and SANFH+TFRD groups was increased whereas the expression of Bcl-2 was decreased in comparison with the control group (Fig. 1D-G; $\mathrm{P}<0.01$ ), while treatment with TFRD increased the expression of Bcl-2 and decreased the expression of Bax and caspase-3 compared with that in the SANFH group (Fig. 1D-G; P<0.01).

TFRD increases the protein expression of VEGF, RUNX2, $O P G$ and $O C N$ and decreases the protein expression of RANKL in the femoral head of SANFH rats. Protein expression in the femoral head of rats was detected via western blotting. 

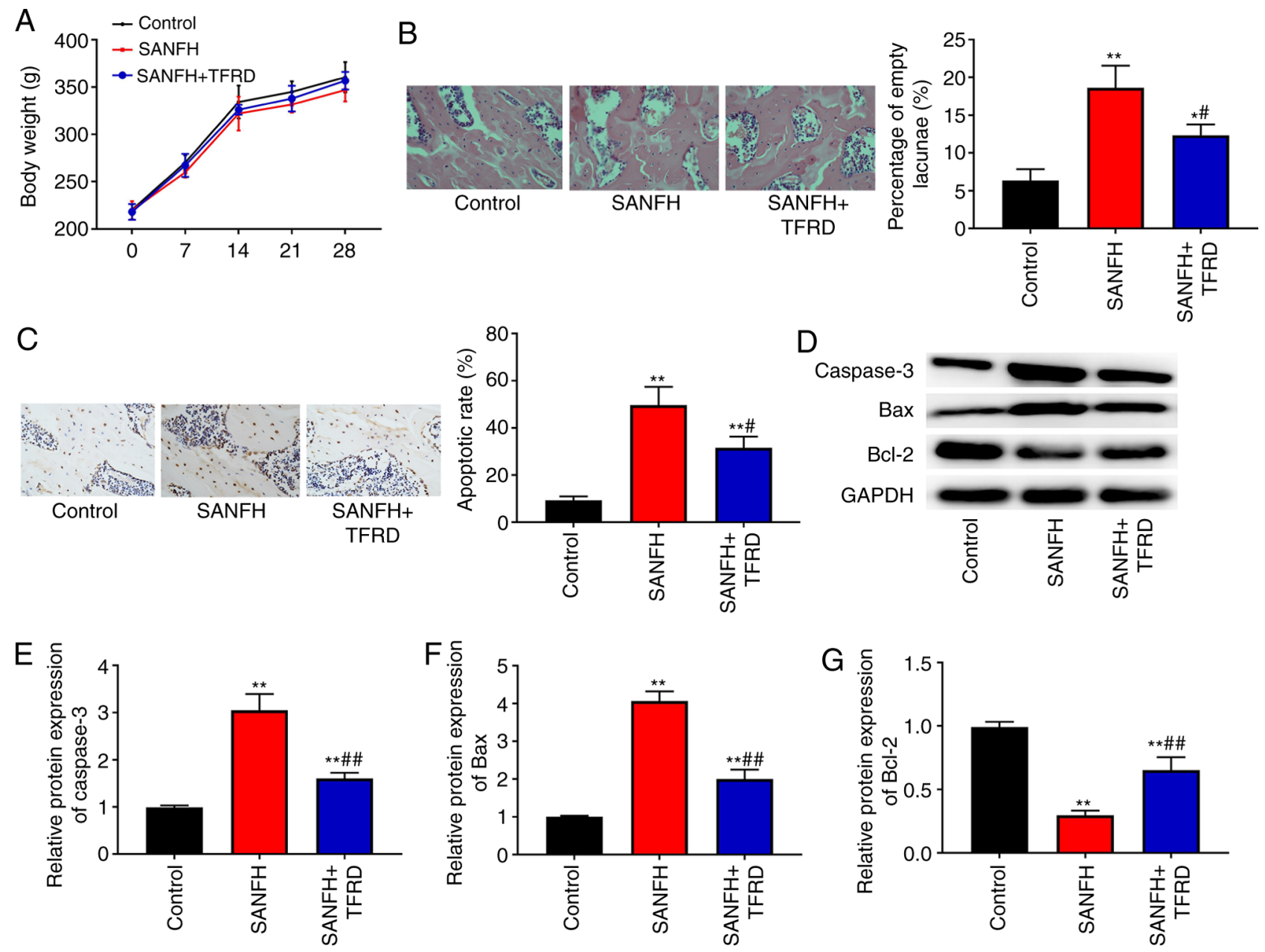

Figure 1. TFRD reduced the pathological changes of the femoral head and inhibited apoptosis of the necrotic zone in SANFH rats. (A) Body weight over time of rats in the control, SANFH and SANFH+TFRD groups $(n=8)$. (B) Haematoxylin and eosin staining was used to assess the pathological changes in the rat femoral head (magnification, x100). (C) TUNEL assay was conducted to detect apoptosis in bone cells of the rat femoral head (magnification, x100). (D) Protein bands of apoptosis related proteins (caspase-3, Bax and Bcl-2). The expression of (E) caspase-3, (F) Bax and (G) Bcl-2 was detected using western blotting. " $\mathrm{P}<0.05$ and ${ }^{* *} \mathrm{P}<0.01$ vs. the control group; ${ }^{*} \mathrm{P}<0.05$ and ${ }^{\# \#} \mathrm{P}<0.01$ vs. the SANFH group. TFRD, total flavonoids from Rhizoma drynariae; SANFH, steroid-induced avascular necrosis of the femoral head.

As shown in Fig. 2, Compared with the control group, the expression of VEGF, RUNX2, OPG and OCN in the SANFH and SANFH+TFRD groups was decreased $(\mathrm{P}<0.01)$, whereas the expression of RANKL was increased $(\mathrm{P}<0.01)$. Compared with the SANFH group, treatment with TFRD increased the expression of VEGF, RUNX2, OPG and OCN and reduced the expression of RANKL $(\mathrm{P}<0.05)$.

TFRD activates the PISK/AKT pathway in the SANFH rats. To explore the protective mechanism of TFRD in SANFH rats, the PI3K/AKT pathway-related proteins were detected by western blotting. The results showed that the SANFH and SANFH+TFRD groups had lower expression of $\mathrm{p}-\mathrm{P} 13 \mathrm{~K} / \mathrm{P} 13 \mathrm{~K}$ and p-AKT/AKT than that in the control group ( $\mathrm{P}<0.01$; Fig. 3), while treatment with TFRD increased the protein expression of $\mathrm{p}-\mathrm{P} 13 \mathrm{~K} / \mathrm{P} 13 \mathrm{~K}$ and $\mathrm{p}-\mathrm{AKT} / \mathrm{AKT}$ compared with the SANFH group ( $\mathrm{P}<0.01$; Fig. 3$)$.

TFRD promotes proliferation, inhibits apoptosis, reduces ROS levels and activates the PI3K/AKT pathway in osteoblasts. The effect of different concentrations of
Dex at different time points on osteoblast proliferation was investigated using MTT assay. The results showed that Dex inhibited osteoblast proliferation in a dose- and time-dependent manner and that $10^{-6} \mathrm{M}$ Dex inhibited osteoblast proliferation $(\mathrm{P}<0.05$; Fig. $4 \mathrm{~A})$. Additionally, MTT assay was used to evaluate the cytotoxic effect of TFRD on osteoblasts. As shown in Fig. 4B, when the concentration of TFRD was less than $12.5 \mu \mathrm{g} / \mathrm{ml}$, TFRD had no cytotoxic effect on osteoblasts. Therefore, $10^{-6} \mathrm{M}$ Dex and $12.5 \mu \mathrm{g} / \mathrm{ml}$ TFRD were selected for subsequent experiments. As can be seen in Fig. 4C-E, treatment with Dex reduced the $\mathrm{OD}_{490}$ value, increased apoptotic rate and ROS production in osteoblasts compared with the control group $(\mathrm{P}<0.01)$, while the opposite effects of TFRD in osteoblasts were observed. Compared with the control group, Dex increased the expression of caspase- 3 and Bax and decreased the expression of Bcl-2, p-P13K/P13K and p-AKT/AKT (P<0.01; Fig. 4F), but this effect was weakened by TFRD.

TFRD protects osteoblasts from Dex-induced damage through the PI3K/AKT pathway. In order to elucidate the mechanism 
A

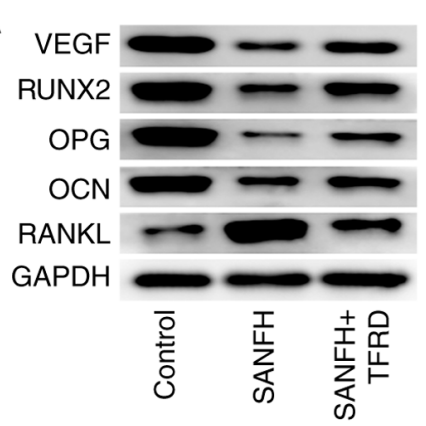

D

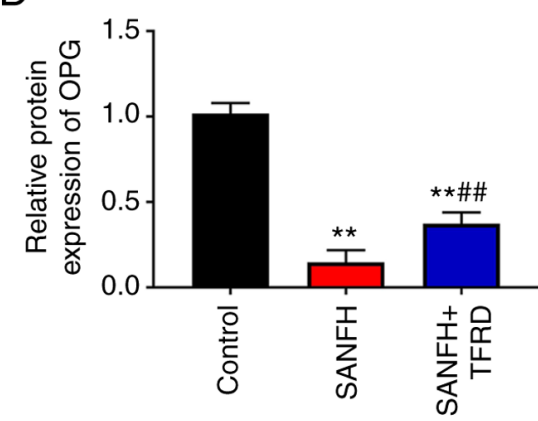

B

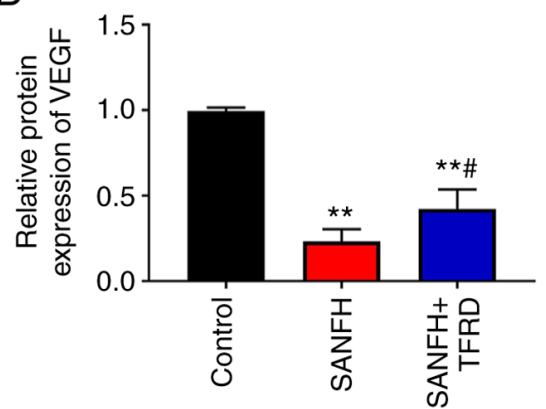

E

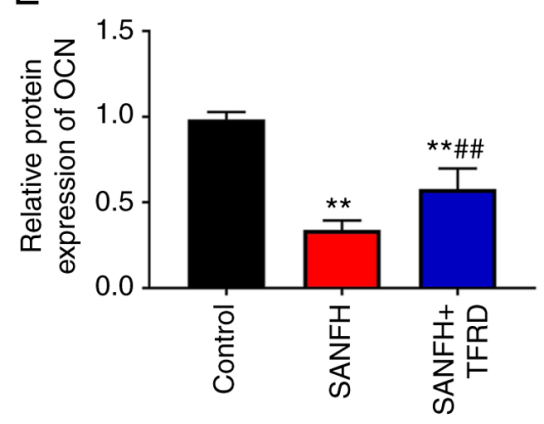

C

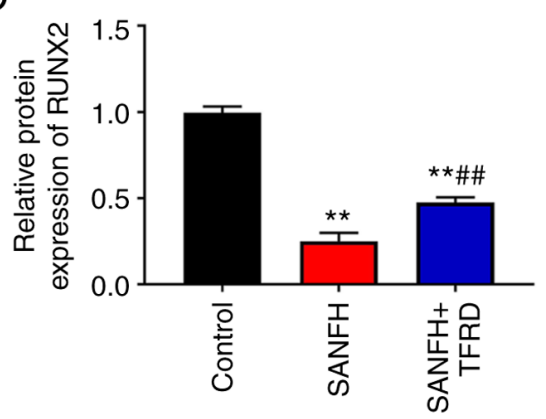

$\mathrm{F}$

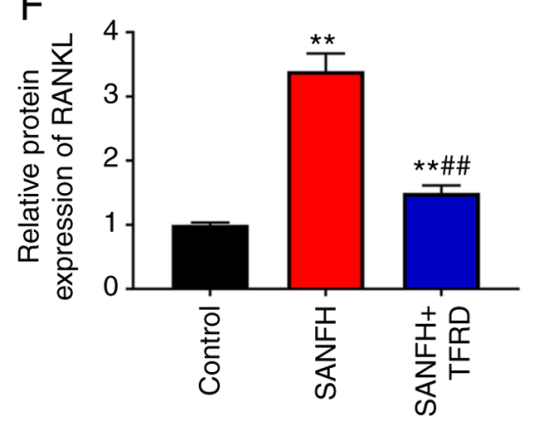

Figure 2. TFRD increased the expression of VEGF, RUNX2, OPG and OCN and decreased the expression of RANKL in the femoral head of SANFH rats. (A) Protein bands of VEGF, RUNX2, OPG, OCN and RANKL. The expression of (B) VEGF, (C) RUNX2, (D) OPG, (E) OCN and (F) RANKL in the femoral head of rats was detected using western blotting. ${ }^{* *} \mathrm{P}<0.01$ vs. the control group; ${ }^{*} \mathrm{P}<0.05$ and ${ }^{\# \#} \mathrm{P}<0.01$ vs. the SANFH group. TFRD, total flavonoids from Rhizoma drynariae; RUNX2, runt-related transcription factor 2; OPG, osteoprotegerin; OCN, osteocalcin; RANKL, receptor activator of nuclear factor kappa B ligand; SANFH, steroid-induced avascular necrosis of the femoral head.
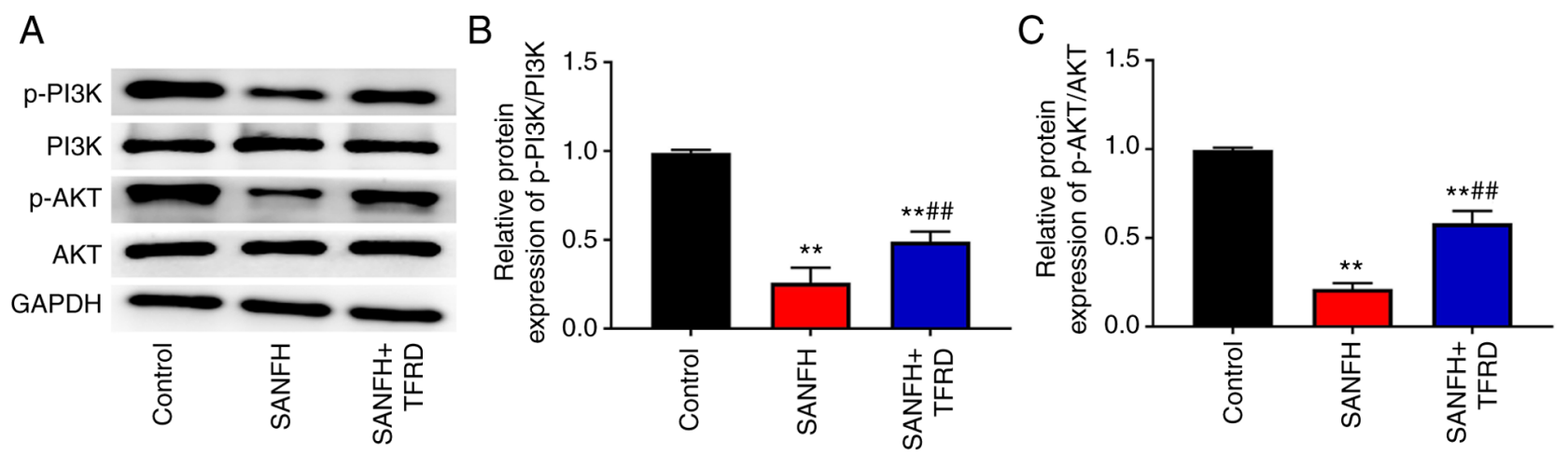

Figure 3. TFRD activated the PI3K/AKT pathway in the SANFH rats. (A) Protein bands of p-PI3K, PI3K, p-AKT and AKT. The expression of p-PI3K/PI3K (B) and p-AKT/AKT (C) in the femoral head of rats was detected using western blotting assay. ${ }^{* *} \mathrm{P}<0.01$ vs. the control group; ${ }^{* \#} \mathrm{P}<0.01$ vs. the SANFH group. TFRD, total flavonoids from Rhizoma drynariae; PI3K, phosphoinositide 3-kinase; SANFH, steroid-induced avascular necrosis of the femoral head; p-, phosphorylated.

of TFRD-induced protection of osteoblasts from Dex-induced damage, the PI3K/AKT pathway inhibitor (LY294002) was used to treat the osteoblasts in the Dex+TFRD group. As shown in Fig. 5A, the expression of $\mathrm{p}-\mathrm{P} 13 \mathrm{~K} / \mathrm{P} 13 \mathrm{~K}$ and $\mathrm{p}-\mathrm{AKT} / \mathrm{AKT}$ in the Dex+TFRD+LY294002 group was reduced compared with that in the Dex+TFRD group $(\mathrm{P}<0.01)$, indicating that the activation of the PI3K/AKT pathway induced by TFRD was blocked via LY294002. Subsequently, the effects of LY294002 on proliferation, apoptosis and ROS production was detected in osteoblasts. As presented in Fig. 5B-D, following LY294002 treatment, the $\mathrm{OD}_{490}$ value was decreased, while the apoptotic rate and ROS production were increased in the osteoblasts $(\mathrm{P}<0.05)$.

\section{Discussion}

ONFH is a common disease, mainly caused by the abuse of steroid hormones (21). SANFH is characterized by difficult recovery and poor prognosis, which has a serious impact on the health and quality of life of patients (22). The main chemical constituents of the Rhizoma drynariae extract include phenolic acids, flavonoids and triterpenes, as well as their glycosides (8). Among these, studies have mainly focused on total flavonoids (23-25). As TFRD has antioxidant properties and anti-osteoporosis activity, it has been widely used in clinic and has great value in research and development (26). The present study found that TFRD reduced the pathological 

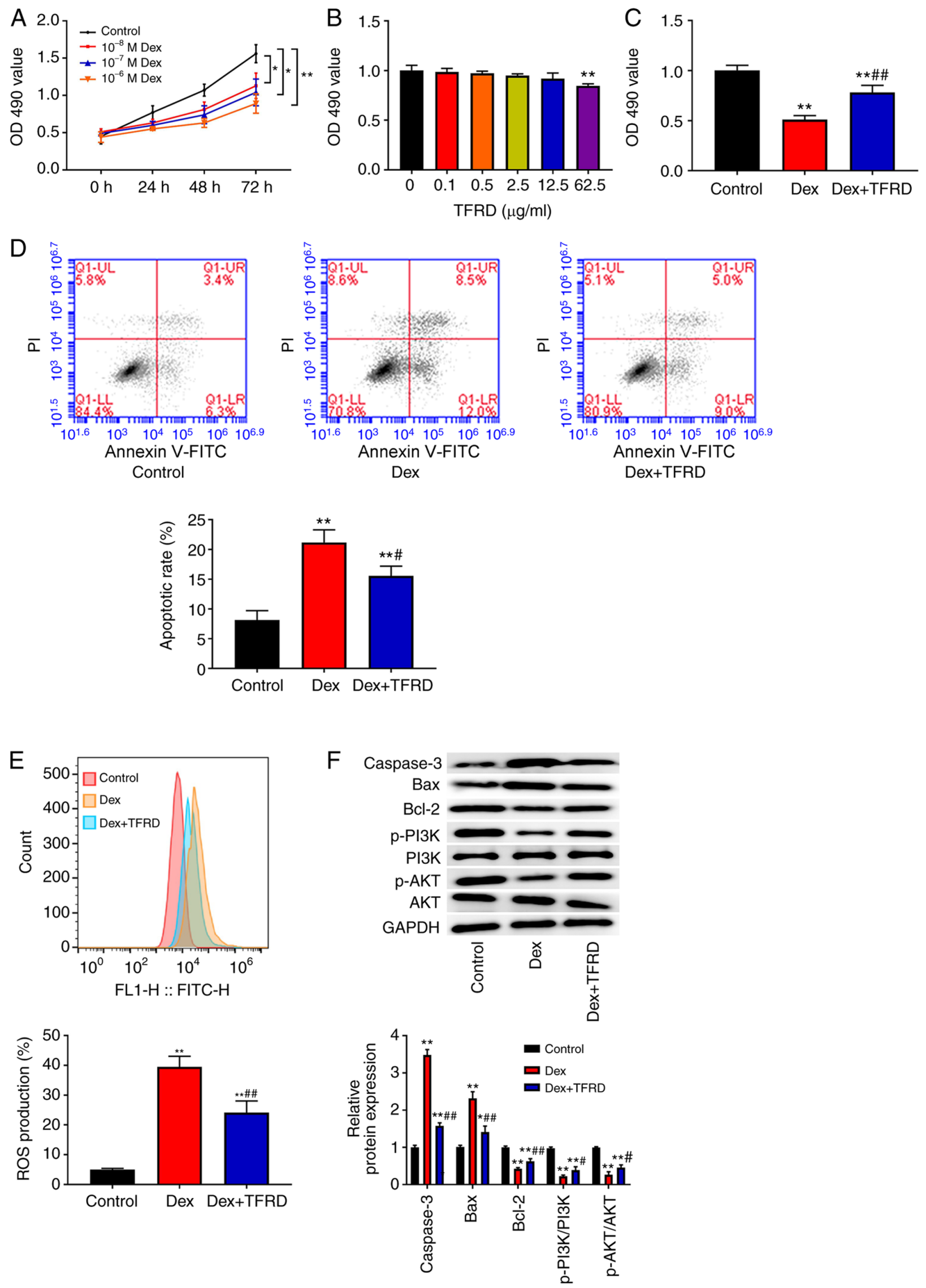

Figure 4. TFRD promoted proliferation, inhibited apoptosis, reduced ROS levels and activated the PI3K/AKT pathway in osteoblast. (A-C) MTT assay was used to detect osteoblast proliferation. (D) Flow cytometry was used to analyse osteoblast apoptosis. (E) DCFH-DA staining was used to detect the ROS level in osteoblasts. (F) The expression of caspase-3, Bax, Bcl-2, p-PI3K/PI3K and p-AKT/AKT in osteoblasts was detected using western blotting. "P $<0.05$ and ${ }^{* *} \mathrm{P}<0.01$ vs. the control group; ${ }^{\#} \mathrm{P}<0.05$ and ${ }^{\# \#} \mathrm{P}<0.01$ vs. the Dex group. TFRD, total flavonoids from Rhizoma drynariae; ROS, reactive oxygen species; PI3K, phosphoinositide 3-kinase; p-, phosphorylated; DCFH-DA, dichlorofluorescein-diacetate; Dex, dexamethasone; OD, optical density; FITC, fluorescein isothiocyanate. 

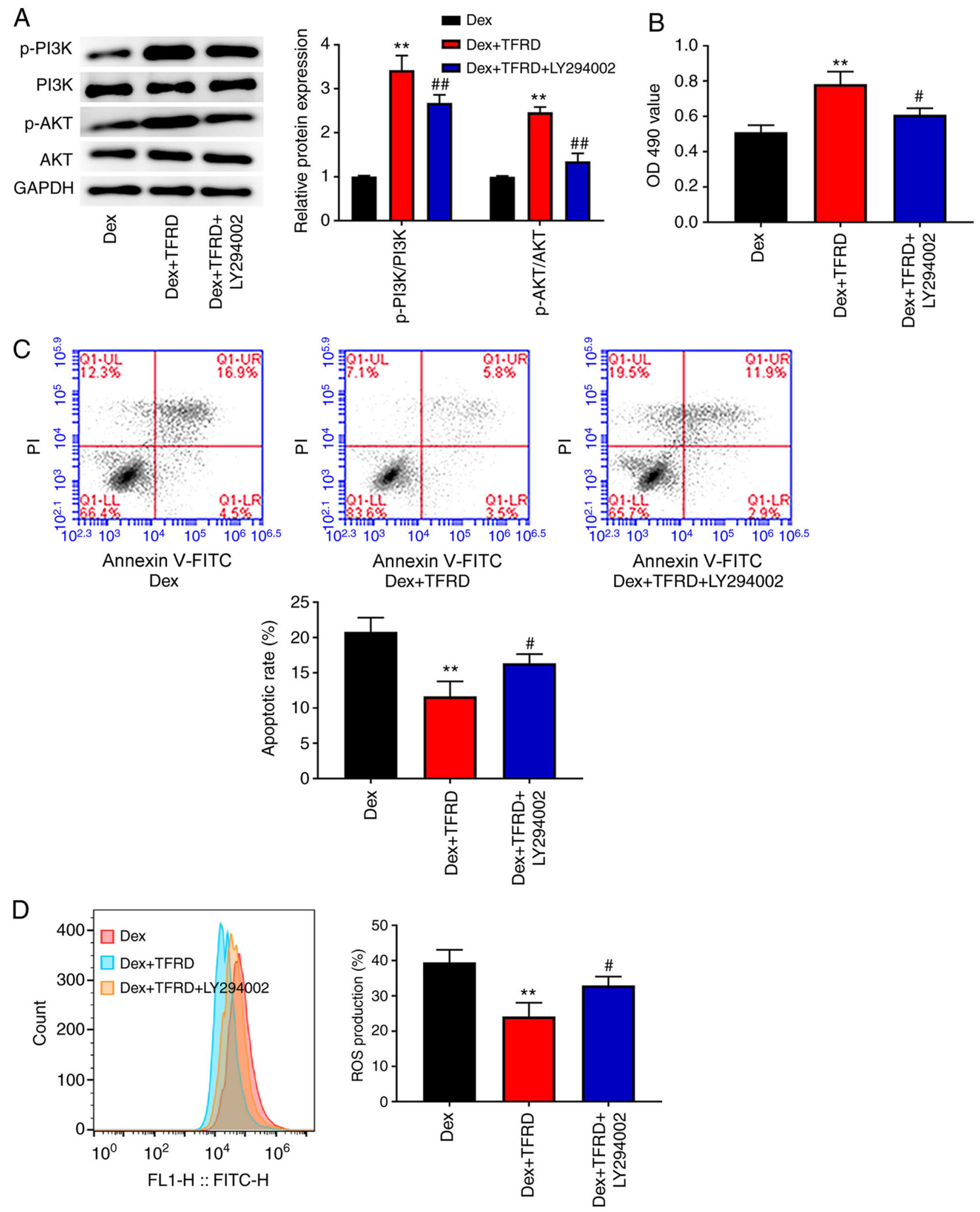

Figure 5. TFRD protected osteoblast from Dex-induced damage through the PI3K/AKT pathway. (A) The expression of p-PI3K/PI3K and p-AKT/AKT in osteoblasts was detected using western blotting. (B) MTT assay was used to detect osteoblast proliferation. (C) Flow cytometry was used to analyse osteoblast apoptosis. (D) DCFH-DA staining was used to detect the ROS level in osteoblast. ${ }^{* *} \mathrm{P}<0.01$ vs. the Dex group; ${ }^{\#} \mathrm{P}<0.05$ and ${ }^{\# \#} \mathrm{P}<0.01$ vs. the Dex+TFRD group. TFRD, total flavonoids from Rhizoma drynariae; Dex, dexamethasone; PI3K, phosphoinositide 3-kinase; p-, phosphorylated; ROS, reactive oxygen species; DCFH-DA, dichlorofluorescein-diacetate.

changes, inhibited cell apoptosis, increased the expression of VEGF, RUNX2, OPG and OCN, decreased RANKL expression and activated the PI3K/AKT pathway in SANFH rats. In addition, TFRD promoted proliferation, inhibited 
apoptosis and reduced ROS levels by activating the PI3K/AKT pathway in osteoblasts.

In the treatment of ONFH, restoring the bone blood and nutrient supply is important for bone healing and bone formation $(27,28)$. When the blood supply is obstructed, it causes ischemia and anoxia of the femoral head, leading to the apoptosis of osteoblasts and ONFH $(29,30)$. VEGF is a crucial angiogenic factor that regulates the proliferation and migration of vascular endothelial cells and vascularization and serves a key role in bone formation and healing (31-33). In addition, VEGF and endothelial cells can induce osteogenic differentiation of bone marrow-derived mesenchymal stem cells (34). The present study found that VEGF expression in rats with SANFH was decreased, while treatment with TFRD increased VEGF expression. These findings indicated that TFRD increases VEGF expression, which may promote bone formation and osteoblast differentiation.

Bone cell damage and the imbalance between osteogenesis and bone exfoliation activities ultimately lead to bone structure destruction and collapse (35). RUNX2, a transcription activator of osteoblasts, serves an important role in the regulation of gene expression during osteogenic differentiation (36). The degree of cell deposition and mineralization can be reflected by the expression level of OCN, which is involved in the late stage of osteogenic differentiation (37). OPG produced by osteoblasts can bind to osteoblast membranes, block the differentiation of osteoclast precursors and promote osteoclast apoptosis, thus preventing the development of ONFH $(38,39)$. The present study detected the expression of RUNX2, OPG, OCN and RANKL in the femoral head of rats. Treatment with TFRD was found to increase the expression of RUNX2, OPG and OCN and reduce the expression of RANKL. The above results indicate that TFRD promotes the differentiation of osteoblasts.

Ischaemic necrosis of the femoral head is the main process resulting in bone cell death; after ischaemia and hypoxia occur, cell death (apoptosis) is activated (40). Previous studies have shown that Dex can induce osteoblast apoptosis and SANFH in rat or human specimens by upregulating caspase-3 $(20,41)$. Bcl-2 is an anti-apoptotic gene, whereas Bax is a pro-apoptotic gene (3) and studies have reported that the cause of Dex-induced osteoblast apoptosis is the imbalance between Bcl-2 and Bax (mitochondrial dysfunction) $(42,43)$. Additionally, Dex treatment can increase ROS production and excessive ROS may cause cell apoptosis through the mitochondrial-mediated caspase apoptosis pathway (44). In the present study, SANFH modelling and Dex treatment promoted cell apoptosis, increased the expression of caspase- 3 and Bax and decreased the protein expression of Bcl-2 in vivo and in vitro. In addition, compared with the SANFH and Dex groups, TFRD treatment inhibited cell apoptosis, decreased the protein expression of caspase- 3 and Bax and increased the protein expression of Bcl-2. TFRD treatment reduced ROS production and promoted Dex-induced osteoblast proliferation. These findings indicate that TFRD serves an anti-apoptosis role in SANFH partly by inhibiting the mitochondrial pathway.

At present, the pathogenesis of ONFH is still unclear, although apoptosis is one of its most studied mechanisms (45). Previous studies have reported apoptotic signals transduced by the $\mathrm{PI} 3 \mathrm{~K} / \mathrm{AKT}-\mathrm{Bax} / \mathrm{Bcl}-2 /$ caspase-3 pathway in some

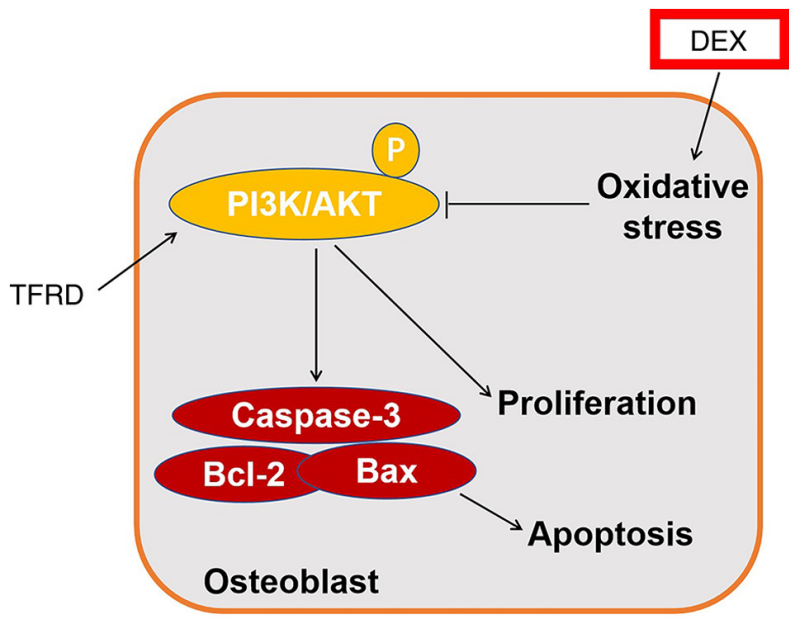

Figure 6. A brief schematic diagram showing the underlying mechanism of TFRD in SANFH. TFRD, total flavonoids from Rhizoma drynariae; SANFH, steroid-induced avascular necrosis of the femoral head; PI3K, phosphoinositide 3-kinase; Dex, dexamethasone; p, phosphorylation.

orthopaedic diseases $(46,47)$. In addition, Cakir et al (48) have found that the accumulation of ROS causes oxidative stress and activates the c-Jun N-terminal kinase pathway, which may inhibit the activation of the AKT pathway and promote osteoblast apoptosis. The present study found that SANFH modelling and Dex treatment reduced the expression of $\mathrm{p}-\mathrm{P} 13 \mathrm{~K} / \mathrm{P} 13 \mathrm{~K}$ and $\mathrm{p}-\mathrm{AKT} / \mathrm{AKT}$ in vivo and in vitro, while treatment with TFRD increased the protein expression of $\mathrm{p}-\mathrm{P} 13 \mathrm{~K} / \mathrm{P} 13 \mathrm{~K}$ and $\mathrm{p}-\mathrm{AKT} / \mathrm{AKT}$ compared with expression in SANFH and Dex groups. The addition of PI3K inhibitor (LY294002) reversed the promotion of the PI3K/AKT pathway in osteoblasts induced by TFRD, as well as the promotion of proliferation and inhibition of apoptosis and ROS production in osteoblasts caused by TFRD. These findings suggested that TFRD protects osteoblasts from Dex-induced damage through the PI3K/AKT pathway (Fig. 6).

In conclusion, TFRD protected against SANFH in a rat model. In addition, TFRD protected osteoblasts from steroidal damage via PI3K/AKT pathway. The findings of the present study may have implications for future treatments of SANFH.

\section{Acknowledgements}

Not applicable.

\section{Funding}

No funding was received.

\section{Availability of data and materials}

The datasets used and analysed during the current study are available from the corresponding author on reasonable request.

\section{Authors' contributions}

WL and QY designed the study; MY, PK and BY performed the experiments, analysed the data and wrote the paper. WL 
and QY confirm the authenticity of all the raw data. All authors reviewed and approved the final manuscript.

\section{Ethics approval and consent to participate}

The experimental protocol of the present study was performed in accordance with the Guide for the Care and Use of Laboratory Animals and approved by the Affiliated Hospital of Shandong University of Traditional Chinese Medicine (approval no. 2019-58).

\section{Patient consent for publication}

Not applicable.

\section{Competing interests}

The authors declare that they have no competing interests.

\section{References}

1. Xiang S, Li Z and Weng X: The role of lncRNA RP11-154D6 in steroid-induced osteonecrosis of the femoral head through BMSC regulation. J Cell Biochem 120: 18435-18445, 2019.

2. Yan YQ, Pang QJ and Xu RJ: Effects of erythropoietin for precaution of steroid-induced femoral head necrosis in rats. BMC Musculoskelet Disord 19: 282, 2018.

3. Xue XH, Feng ZH, Li ZX and Pan XY: Salidroside inhibits steroid-induced avascular necrosis of the femoral head via the $\mathrm{PI} 3 \mathrm{~K} / \mathrm{Akt}$ signaling pathway: In vitro and in vivo studies. Mo Med Rep 17: 3751-3757, 2018.

4. Ren X, Fan W, Shao Z, Chen K, Yu X and Liang Q: A metabolomic study on early detection of steroid-induced avascular necrosis of the femoral head. Oncotarget 9: 7984-7995, 2018.

5. Wong GKC, Poon WS and Chiu KH: Steroid-induced avascular necrosis of the hip in neurosurgical patients: Epidemiological study. ANZ J Surg 75: 409-410, 2015.

6. Bae WY, Kim HY, Choi KS, Chang KH, Hong YH, Eun J, Lee NK and Paik HD: Investigation of Brassica juncea, Forsythia suspensa, and Inula britannica: Phytochemical properties, antiviral effects, and safety. BMC Complement Altern Med 19: 253, 2019.

7. Li L, Hou X, Xu R, Liu C and Tu M: Research review on the pharmacological effects of astragaloside IV. Fundam Clin Pharmacol 31: 17-36, 2017.

8. Zhang Y, Jiang J, Shen H, Chai Y, Wei X and Xie Y: Total flavonoids from Rhizoma Drynariae (Gusuibu) for treating osteoporotic fractures: Implication in clinical practice. Drug Des Devel Ther 11: 1881-1890, 2017.

9. Lin FX, Du SX, Liu DZ, Hu QX, Yu GY, Wu CC, Zheng GZ, Xie D, Li XD and Chang B: Naringin promotes osteogenic differentiation of bone marrow stromal cells by up-regulating Foxc2 expression via the IHH signaling pathway. Am J Trans Res 8: 5098-5107, 2016

10. Wong RW, Rabie B, Bendeus M and Hägg U: The effects of rhizoma curculiginis and rhizoma drynariae extracts on bones. Chin Med 2: 13, 2007.

11. Kuang MJ, Zhang WH, He WW, Sun L, Ma JX, Wang D and Ma XL: Naringin regulates bone metabolism in glucocorticoid-induced osteonecrosis of the femoral head via the Akt/Bad signal cascades. Chem Biol Interact 304: 97-105, 2019.

12. Guntur AR and Rosen CJ: The skeleton: A multi-functional complex organ. New insights into osteoblasts and their role in bone formation: The central role of PI3Kinase. J Endocrinol 211: 123-130, 2011

13. Gu Y, Du J, Si M, Mo J, Qiao S and Lai H: The roles of PI3K/Akt signaling pathway in regulating MC3T3-E1 preosteoblast proliferation and differentiation on SLA and SLActive titanium surfaces. J Biomed Mater Res A 101: 748-754, 2013.

14. Ma J, Sawai H, Ochi N, Matsuo Y, Xu D, Yasuda A, Takahashi H, Wakasugi $\mathrm{T}$ and Takeyama $\mathrm{H}$ : PTEN regulates angiogenesis through PI3K/Akt/VEGF signaling pathway in human pancreatic cancer cells. Mol Cell Biochem 331: 161-171, 2009.
15. Zhang J, Liu X, Li H, Chen C, Hu B, Niu X, Li Q, Zhao B, Xie Z and Wang Y: Exosomes/tricalcium phosphate combination scaffolds can enhance bone regeneration by activating the PI3K/Akt signaling pathway. Stem Cell Res Ther 7: 136, 2016.

16. Hu J, Gu Y and Fan W: Rg1 protects rat bone marrow stem cells against hydrogen peroxide-induced cell apoptosis through the PI3K/Akt pathway. Mol Med Rep 14: 406-412, 2016.

17. National Research Council: Guide for the Care and Use of Laboratory Animals: Eighth edition. The National Academies Press, Washington, DC, 2011.

18. Spiers DE and Candas V: Relationship of skin surface area to body mass in the immature rat: A reexamination. J Appl Physiol Respir Environ Exerc Physiol 56: 240-243, 1984.

19. Lu X, Xiong Z, Li J, Zheng S, Huo T and Li F: Metabonomic study on 'Kidney-Yang Deficiency syndrome' and intervention effects of Rhizoma Drynariae extracts in rats using ultra performance liquid chromatography coupled with mass spectrometry. Talanta 83: 700-708, 2011.

20. Feng Z, Zheng W, Tang Q, Cheng L, Li H, Ni W and Pan X: Fludarabine inhibits STAT1-mediated up-regulation of caspase-3 expression in dexamethasone-induced osteoblasts apoptosis and slows the progression of steroid-induced avascular necrosis of the femoral head in rats. Apoptosis 22: 1001-1012, 2017.

21. Wang A, Ren M and Wang J: The pathogenesis of steroid-induced osteonecrosis of the femoral head: A systematic review of the literature. Gene 671: 103-109, 2018.

22. Song HM, Wei YC, Li N, Wu B, Xie N, Zhang KM, Wang SZ and Wang HM: Effects of Wenyangbushen formula on the expression of VEGF, OPG, RANK and RANKL in rabbits with steroid-induced femoral head avascular necrosis. Mol Med Rep 12: 8155-8161, 2015.

23. Xu L, Huang G, Guo X, Zhou Q and He S: Total flavonoids, extracted from Polygonum knotweed L, exert beneficial hepatoprotection against liver injury. J Cell Biochem 120: 12677-12683, 2019.

24. Cheng Y, Yang Z, Shi J, Yang J, Zhao J, He Y and Qi M: Total flavonoids of Epimedium ameliorates testicular damage in streptozotocin-induced diabetic rats by suppressing inflammation and oxidative stress. Environ Toxicol 35: 268-276, 2020.

25. Guo Y, Xing L, Chen N, Gao C, Ding Z and Jin B: Total flavonoids from the Carya cathayensis Sarg. leaves inhibit HUVEC senescence through the miR-34a/SIRT1 pathway. J Cell Biochem 120: 17240-17249, 2019.

26. Kang SN, Lee JS, Park JH, Cho JH, Park JH, Cho KK, Lee OH and Kim IS: In vitro anti-osteoporosis properties of diverse Korean Drynariae rhizoma phenolic extracts. Nutrients 6: $1737-1751,2014$

27. Weinstein RS: Glucocorticoid-induced osteonecrosis Endocrine 41: 183-190, 2012

28. Assouline-Dayan Y, Chang C, Greenspan A, Shoenfeld Y and Gershwin ME: Pathogenesis and natural history of osteonecrosis. Semin Arthritis Rheum 32: 94-124, 2002.

29. Inoue A and Ono K: A histological study of idiopathic avascular necrosis of the head of the femur. J Bone Joint Surg Br 61-B: 138-143, 1979.

30. Ichiseki T, Ueda S, Ueda Y, Tuchiya M, Kaneuji A and Kawahara N: Involvement of necroptosis, a newly recognized cell death type, in steroid-induced osteonecrosis in a rabbit model. Int J Med Sci 14: 110-114, 2017.

31. Varoga D, Drescher W, Pufe M, Groth G and Pufe T: Differential expression of vascular endothelial growth factor in glucocorticoid-related osteonecrosis of the femoral head. Clin Orthop Relat Res 467: 3273-3282, 2009.

32. Carlevaro MF, Cermelli S, Cancedda R and Cancedda FD: Vascular endothelial growth factor (VEGF) in cartilage neovascularization and chondrocyte differentiation: Auto-paracrine role during endochondral bone formation. J Cell Sci 113: 59-69, 2000.

33. Carmeliet P, Ferreira V, Breier G, Pollefeyt S, Kieckens L, Gertsenstein M, Fahrig M, Vandenhoeck A, Harpal K, Eberhardt C, et al: Abnormal blood vessel development and lethality in embryos lacking a single VEGF allele. Nature 380: 435-439, 1996.

34. Zheng LZ, Cao HJ, Chen SH, Tang T, Fu WM, Huang L, Chow DHK, Wang YX, Griffith JF, He W, et al: Blockage of src by specific siRNA as a novel therapeutic strategy to prevent destructive repair in steroid-associated osteonecrosis in rabbits. J Bone Miner Res 30: 2044-2057, 2015. 
35. Piuzzi NS, Anis HK and Muschler GF: Osteonecrosis of the femoral head with subchondral collapse. Cleve Clin J Med 86: 511-512, 2019.

36. Zhu C, Lv Y, Qian C, Qian H, Jiao T, Wang L and Zhang F: Proliferation and osteogenic differentiation of rat BMSCs on a novel $\mathrm{Ti} / \mathrm{SiC}$ metal matrix nanocomposite modified by friction stir processing. Sci Rep 6: 38875, 2016.

37. Qian C, Zhu C, Yu W, Jiang $\mathrm{X}$ and Zhang F: High-fat diet/low-dose streptozotocin-induced type 2 diabetes in rats impacts osteogenesis and wnt signaling in bone marrow stromal cells. PLoS One 10: e0136390, 2015.

38. Croucher PI, Mcdonald MM and Martin TJ: Bone metastasis: The importance of the neighbourhood. Nat Rev Cancer 16 373-386, 2016

39. Peng WX, Ye C, Dong WT, Yang LL, Wang CQ, Wei ZA, Wu JH, Li Q, Deng J and Zhang J: MicroRNA-34a alleviates steroid-induced avascular necrosis of femoral head by targeting Tgif2 through OPG/RANK/RANKL signaling pathway. Exp Biol Med (Maywood) 242: 1234-1243, 2017.

40. Zhang H, Zhou F, Pan Z, Bu X, Wang Y and Chen F. $11 \beta$-hydroxysteroid dehydrogenases- 2 decreases the apoptosis of MC3T3/MLO-Y4 cells induced by glucocorticoids. Biochem Biophys Res Commun 490: 1399-1406, 2017.

41. Xu X, Wen $\mathrm{H}, \mathrm{Hu} \mathrm{Y}, \mathrm{Yu} \mathrm{H}$, Zhang Y, Chen $\mathrm{C}$ and Pan $\mathrm{X}$ STAT1-caspase 3 pathway in the apoptotic process associated with steroid-induced necrosis of the femoral head. J Mol Histol 45: 473-485, 2014.

42. Li DW, Liu ZQ, Chen W, Yao M and Li GR: Association of glycogen synthase kinase- $3 \beta$ with Parkinson's disease (Review) Mol Med Rep 9: 2043-2050, 2014.
43. Chao DT and Korsmeyer SJ: BCL-2 family: Regulators of cell death. Annu Rev Immunol 16: 395-419, 1998.

44. Ravindran J, Gupta N, Agrawal M, Asb B and Rao PVL: Modulation of ROS/MAPK signaling pathways by okadaic acid leads to cell death via, mitochondrial mediated caspase-dependent mechanism. Apoptosis 16: 145-161, 2011.

45. Deng S, Zhou JL, Fang HS, Nie ZG, Chen S and Peng H: Sesamin protects the femoral head from osteonecrosis by inhibiting ROS-induced osteoblast apoptosis in rat model. Front Physiol 9: 1787,2018

46. Sato A, Tu X, McAndrews K, Plotkin L and Bellido T: Prevention of glucocorticoid induced-apoptosis of osteoblasts and osteocytes by protecting against endoplasmic reticulum (ER) stress in vitro and in vivo in female mice. Bone 73: 60-68, 2015.

47. Li J, He C, Tong W, Zou Y, Li D, Zhang C and Xu W: Tanshinone IIA blocks dexamethasone-induced apoptosis in osteoblasts through inhibiting Nox4-derived ROS production. Int J Clin Exp Pathol 8: 13695-13706, 2015

48. Cakir E, Yilmaz A, Demirag F, Oguztuzun S, Sahin S, Yazici UE and Aydin M: Prognostic significance of micropapillary pattern in lung adenocarcinoma and expression of apoptosis-related markers: Caspase-3, bcl-2, and p53. APMIS 119: 574-580, 2011.

This work is licensed under a Creative Commons Attribution-NonCommercial-NoDerivatives 4.0 International (CC BY-NC-ND 4.0) License. 